\title{
Signatures of Surface Targets with Increasing Speed in Spotlight Synthetic Aperture Radar
}

\author{
David Alan Garren \\ Electrical and Computer Engineering Department \\ Naval Postgraduate School \\ Monterey, California 93943, USA \\ Email: dagarren@nps.edu
}

\begin{abstract}
This paper investigates the signature characteristics in spotlight synthetic aperture radar (SAR) imagery for surface targets that are increasing speed during the collection interval. This study considers the broadside geometry case wherein the radar sensor moves with constant speed and heading on a level flight path. The analysis concentrates on the target migration effects in which the moving target smear exhibits some defocus in the range direction, although much smaller in magnitude than the smearing in the radar cross-range direction. This paper focuses on the case of a target that executes a speed transition during the SAR collection interval. The SAR simulations are shown to give moving target signatures that are in agreement with the predicted shapes of the central contours.
\end{abstract}

\section{INTRODUCTION}

Moving targets cause smeared signatures within SAR imagery for all imaging modes. This effect is well-known and has been studied by numerous researchers. For example, Perry et al. [1] develops techniques for generated refocused target signatures based upon the smeared signatures of moving targets. These techniques assume that the smearing lies entirely in the radar cross-range direction, as has been assumed with most other studies. In addition, a number of other researchers have studied the properties of moving target signatures within SAR imagery [2].

For narrow-band SAR, the signature lies almost entirely in the radar cross-range direction. However, for cases in which the radar bandwidth is a significant fraction of the center frequency, the mover signature can exhibit smearing effects in the radar down-range direction as well. Jao [3] has presented the results that moving target signatures can exhibit a basic curved bowing shape, wherein the majority of the smearing still lies in the radar cross-range direction. This bowing can be either concave up or concave down, depending upon the details of the target heading and the platform motion. These curved signatures have often been called target migration effects. These target migration effects are of interest in attempting to understand the motion of surface targets based upon their signatures within SAR imagery.

Stolt [4] has developed techniques for compensating the effects of range migration through the use of the special interpolation methods. These techniques can be applied in the focusing of stationary scenes for low frequency radar data via the Range Migration Algorithm (RMA), even though these methods were originally developed for the analysis of seismic data. The RMA methodology inputs the original smeared signatures of scenes, wherein stationary scattering centers are defocused along curved arcs. These methods then focus these curved arcs to be points for the case of idealized point scattering. Other focusing methods correct only the crossrange defocus effects. Thus, they do not correct residual range migration effects, resulting in lower quality imagery with residual defocus at lower radar frequencies. Other researchers have investigated methods for focusing targets for which range migration effects have caused down-range smearing in the SAR signatures. For example, Rahman [5] has applied RMA in attempting to focus moving targets which have the standard bowing signature presented by Jao [3].

Recent studies [6] examines the signature characteristics of moving targets in SAR image data for cases of non-uniform target motion. Specifically, this analysis considers the case in which a target moves with constant heading at a slower speed, then increases its speed to a higher value. This analysis reveals that complicated smear signatures can occur under these circumstances, even for the special case in which the radar moves with constant speed along a level flight path while collecting SAR measurement data using a broadside imaging geometry. This analysis considers such range migration effects for spotlight-mode SAR, which is an imaging mode [7], [8] that yields a high image resolution at the expense of lower area coverage rate.

An investigation of the morphology of mover smears for general maneuvering targets is given in Ref. [9]. This analysis utilizes power series expansion methods applied to local subaperture SAR images in order to generate general analytic equations for the shape of surface mover smears. Specifically, this study analyzes spotlight SAR for broadside imaging geometry and straight and level radar flight path in order to compute analytic non-parametric expressions for smear signature shapes for surface targets with arbitrary motion profiles. It is possible that this analysis could yield additional insight into the target motion characteristics based upon the details of these smear signatures within SAR imagery.

The fundamental analytics in the previous analysis [9] is based upon the development of analytic equations for the central contour shape for the signature induced by a moving target within SAR sub-aperture images. The use of subaperture SAR images has been applied by various researchers [10], [11], [12] for multiple purposes. The structure of the full-aperture SAR image can be inferred from the details of the sub-aperture images, since the concatenation of the smaller smears of the sub-aperture images yields the full-aperture smear. 
Previous analyses [9], [13] demonstrate the viability and utility of the predictive equations for the central contour shape of the smear for a number of special cases. One of these examples is the case of a surface target that is moving with a constant heading while transitioning from a higher speed to a lower value. The current analysis considers the opposite case wherein a target with constant heading transitions from a slower speed to a higher one. In addition, the current analysis examines the effects on the predicted and actual SAR mover signatures of varying the rate at which the speed increases. To accomplish this goal, free parameters in the analytic predictive equations are varied and the resulting plots are analyzed.

\section{IMAGE FORMATION}

The radar transmits and receives a waveform for each point along the synthetic aperture. The radar receiver forms the complex $I$ and $Q$ channels for each transmitted waveform along the synthetic aperture. The complex phase history data are formed from these two channels, including the magnitude via the complex magnitude of the two channels and the phase via the arctan of the ratio of $Q$ over $I$. These phase history data are measured at the various frequencies $f_{m^{\prime}}$ spanned by the radar waveforms and at the different times $t$ along the synthetic aperture. These operations give the downconverted, frequency-domain SAR measurement data in the original "polar" format:

$$
\tilde{G}\left(f_{m^{\prime}}, t_{n^{\prime}}\right)=\Sigma_{i} \sigma_{i} \exp \left(-j 2 \pi \Delta R_{i}\left(t_{n^{\prime}}\right) 2 f_{m^{\prime}} / c\right) .
$$

In this equation, the path difference relative to the ground reference point (GRP) at the center of the geophysical scene to be imaged is defined by

$$
\Delta R_{i}\left(t_{n^{\prime}}\right) \equiv R_{i}\left(\theta\left(t_{n^{\prime}}\right), \varphi\left(t_{n^{\prime}}\right)\right)-R_{0}\left(\theta\left(t_{n^{\prime}}\right), \varphi\left(t_{n^{\prime}}\right)\right) .
$$

The polar-formatted frequency-domain data are related to collected phase history data by a one-dimensional (1-D) Fourier transform along the range dimension.

The complex-valued measured quantities $\tilde{G}$ in (1) determine the real (i.e., in phase) and imaginary (i.e., quadrature) components of the sub-band centered on frequency sample value $f_{m^{\prime}}$ corresponding to the transmitted radar waveform received at slow-time sample value $t_{n^{\prime}}$. The constant $c$ is the speed of light, and $j=\sqrt{-1}$ is the imaginary constant. The factor of 2 provides for the two-way propagation of the radar. The summation over $i$ in (1) allows for the effects of multiple scattering centers in extended targets. The quantity $R_{i}\left(\theta\left(t_{n^{\prime}}\right), \varphi\left(t_{n^{\prime}}\right)\right)$ is equal to the distance from the radar platform for the waveform received at slow-time $t_{n^{\prime}}$ to the $i$ th scattering center characterized by the complex-valued reflectivity $\sigma_{i}$ :

$$
\begin{aligned}
& R_{i}\left(\theta\left(t_{n^{\prime}}\right), \varphi\left(t_{n^{\prime}}\right)\right) \\
& =\sqrt{\left\{X\left(t_{n^{\prime}}\right)-\alpha\left(t_{n^{\prime}}\right)\right\}^{2}+\left\{Y\left(t_{n^{\prime}}\right)-\beta\left(t_{n^{\prime}}\right)\right\}^{2}+\left\{Z\left(t_{n^{\prime}}\right)\right\}^{2}}
\end{aligned}
$$

Likewise, $R_{0}\left(\theta\left(t_{n^{\prime}}\right), \varphi\left(t_{n^{\prime}}\right)\right)$ equals the distance from the radar platform to the GRP at the $\{x, y, z\}=\{0,0,0\}$ coordinate origin, i.e.,

$$
R_{0}\left(\theta\left(t_{n^{\prime}}\right), \varphi\left(t_{n^{\prime}}\right)\right)=\sqrt{\left\{X\left(t_{n^{\prime}}\right)\right\}^{2}+\left\{Y\left(t_{n^{\prime}}\right)\right\}^{2}+\left\{Z\left(t_{n^{\prime}}\right)\right\}^{2}} .
$$

The image data in the ground plane of $z=0$ are obtained using the following functions that delineate the ground range and the ground cross-range components of the spatial frequency, i.e.,

$$
\begin{aligned}
& \xi_{m}\left(f_{m^{\prime}}, t_{n^{\prime}}\right) \equiv \frac{2 f_{m^{\prime}}}{c} \cos \left(\theta\left(t_{n^{\prime}}\right)\right) \cos \left(\varphi\left(t_{n^{\prime}}\right)\right), \\
& \eta_{m}\left(f_{m^{\prime}}, t_{n^{\prime}}\right) \equiv \frac{2 f_{m^{\prime}}}{c} \cos \left(\theta\left(t_{n^{\prime}}\right)\right) \sin \left(\varphi\left(t_{n^{\prime}}\right)\right) .
\end{aligned}
$$

A polar-to-rectangle sampling operation [7] can be applied to obtain the Cartesian-sampled data, i.e.,

$$
\tilde{G}\left(f_{m^{\prime}}, t_{n^{\prime}}\right) \underset{\text { pol }}{\rightarrow} G\left(\xi_{m}, \eta_{n}\right) .
$$

Here, $G\left(\xi_{m}, \eta_{n}\right)$ is the desired complex-valued frequencydomain Cartesian data. Here, there are $M$ discrete resampled values of the ground range spatial frequency $\xi_{m}$, and there are $N$ discrete resampled values of the ground cross-range spatial frequency $\eta_{n}$. Then, a 2D discrete Fourier transform (DFT) gives the desired spotlight-mode SAR image:

$$
G\left(\xi_{m}, \eta_{n}\right) \underset{2 \mathrm{D} \mathrm{DFT}}{\overleftrightarrow{\longrightarrow}} b\left(x_{k}, y_{\ell}\right)
$$

\section{Signature Predictions}

Ref. [9] applies the techniques of a power series expansion applied to sub-aperture images in order to derive general expressions for the central contour of the smear signature for a surface target moving with an arbitrary motion profile. The present investigation considers the case of a surface target with increasing speed during the SAR collection interval in more detail. Such a maneuvering target can be modeled as a target that moves with constant heading according to a hyperbolic tangent speed profile, i.e., $\tanh (t)$. The integral of this speed profile gives a position profile that is proportional to $\ln (\cosh (t))$. The following form of the true target trajectory is considered [9]:

$$
\begin{aligned}
& \alpha(t)=\bar{\alpha}_{0}+\cos \left(\bar{\phi}_{0}\right)\left\{\bar{v}_{0} t+\bar{w}_{0} \bar{\gamma}_{0} \ln \left(\cosh \left(\frac{t-\bar{t}_{0}}{\bar{\gamma}_{0}}\right)\right)\right\}, \\
& \beta(t)=\bar{\beta}_{0}+\sin \left(\bar{\phi}_{0}\right)\left\{\bar{v}_{0} t+\bar{w}_{0} \bar{\gamma}_{0} \ln \left(\cosh \left(\frac{t-\bar{t}_{0}}{\bar{\gamma}_{0}}\right)\right)\right\},
\end{aligned}
$$

in terms of the free parameters $\left\{\bar{\alpha}_{0}, \bar{\beta}_{0}, \bar{v}_{0}, \bar{w}_{0}, \bar{\gamma}_{0}, \bar{\phi}_{0}, \bar{t}_{0}\right\}$. The current analysis examines the effects of varying the rate at which the target increases speed, as modeled by the parameter $\bar{\gamma}_{0}$. The target speed profile corresponding to (9) can be expressed in the form:

$$
\sqrt{\left[\frac{d \alpha}{d t}\right]^{2}+\left[\frac{d \beta}{d t}\right]^{2}}=\bar{v}_{0}+\bar{w}_{0} \tanh \left(\frac{t-\bar{t}_{0}}{\bar{\gamma}_{0}}\right) .
$$

These equations model a target that is moving at a lower speed and then travels faster, before finally settling at a higher value.

The detailed analytics of Ref. [9] yield the following form for the predicted SAR signature of a target with increasing 


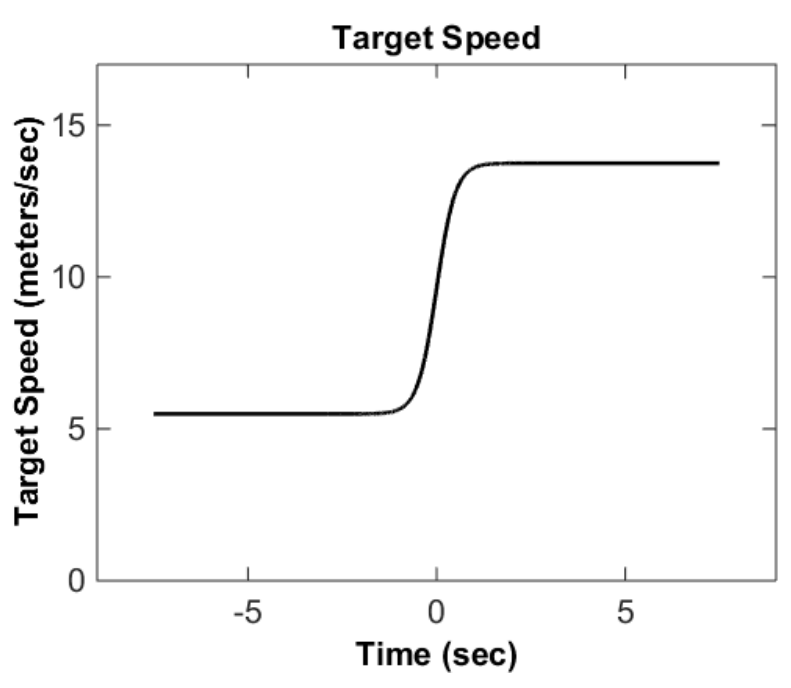

Fig. 1. Speed profile for a target that is increasing in speed according to the motion parameters $\left\{\bar{\alpha}_{0}=0.0 \mathrm{~m}\right\},\left\{\bar{\beta}_{0}=0.0 \mathrm{~m}\right\},\left\{\bar{v}_{0}=9.62 \mathrm{~m} / \mathrm{s}\right\}$, $\left\{\bar{w}_{0}=4.13 \mathrm{~m} / \mathrm{s}\right\},\left\{\bar{\gamma}_{0}=0.5 \mathrm{~s}\right\}$, and $\left\{\bar{\phi}_{0}=-81.3^{\circ}\right\}$

speed, expressed in terms of the down-range and cross-range components:

$$
\begin{aligned}
x\left(\tau_{s}\right) & =\bar{\alpha}_{0}-\tau_{s}^{2} \sin \left(\bar{\phi}_{0}\right) \frac{\bar{v}_{0}}{\kappa_{0}} \\
& +\cos \left(\bar{\phi}_{0}\right) \bar{w}_{0} \bar{\gamma}_{0} \ln \left(\cosh \left(\frac{\tau_{s}-\bar{t}_{0}}{\bar{\gamma}_{0}}\right)\right) \\
& -\tau_{s} \bar{w}_{0}\left\{\cos \left(\bar{\phi}_{0}\right)+\frac{\tau_{s}}{\kappa_{0}} \sin \left(\bar{\phi}_{0}\right)\right\} \tanh \left(\frac{\tau_{s}-\bar{t}_{0}}{\bar{\gamma}_{0}}\right) \\
y\left(\tau_{s}\right) & =\bar{\beta}_{0}+\bar{v}_{0}\left\{\kappa_{0} \cos \left(\bar{\phi}_{0}\right)+\tau_{s} \sin \left(\bar{\phi}_{0}\right)\right\} \\
& +\sin \left(\bar{\phi}_{0}\right)\left\{\bar{v}_{0} \tau_{s}+\bar{w}_{0} \bar{\gamma}_{0} \ln \left(\cosh \left(\frac{\tau_{s}-\bar{t}_{0}}{\bar{\gamma}_{0}}\right)\right)\right\} \\
& +\bar{w}_{0}\left\{\kappa_{0} \cos \left(\bar{\phi}_{0}\right)+\tau_{s} \sin \left(\bar{\phi}_{0}\right)\right\} \tanh \left(\frac{\tau_{s}-\bar{t}_{0}}{\bar{\gamma}_{0}}\right) .
\end{aligned}
$$

Again, the current analysis examines the effects of varying a subset of the parameters within $\left\{\bar{\alpha}_{0}, \bar{\beta}_{0}, \bar{v}_{0}, \bar{w}_{0}, \bar{\gamma}_{0}, \bar{\phi}_{0}, \bar{t}_{0}\right\}$.

\section{Signature Results}

This section examines the specific signatures that result for the case of a moving surface target undergoing an increase in speed during the SAR collection time. This increase in the target speed in modeled through the use of a hyperbolic tangent speed profile as provided in (9)-(10). These truth equations for the actual target motion are consistent with a speed profile of (11), as plotted in Figure 1. For the first example, the parameter value are selected to be $\left\{\bar{\alpha}_{0}=0.0 \mathrm{~m}\right\},\left\{\bar{\beta}_{0}=0.0 \mathrm{~m}\right\}$, $\left\{\bar{v}_{0}=9.62 \mathrm{~m} / \mathrm{s}\right\},\left\{\bar{w}_{0}=4.13 \mathrm{~m} / \mathrm{s}\right\},\left\{\bar{\gamma}_{0}=0.5 \mathrm{~s}\right\}$, and $\left\{\bar{\phi}_{0}=-81.3^{\circ}\right\}$. This set of target motion parameters is consistent with the desired case in which a constant-heading target is initially moving with approximately constant speed and then undergoes an increase in speed, and then settles to a higher approximately constant speed with the same heading.

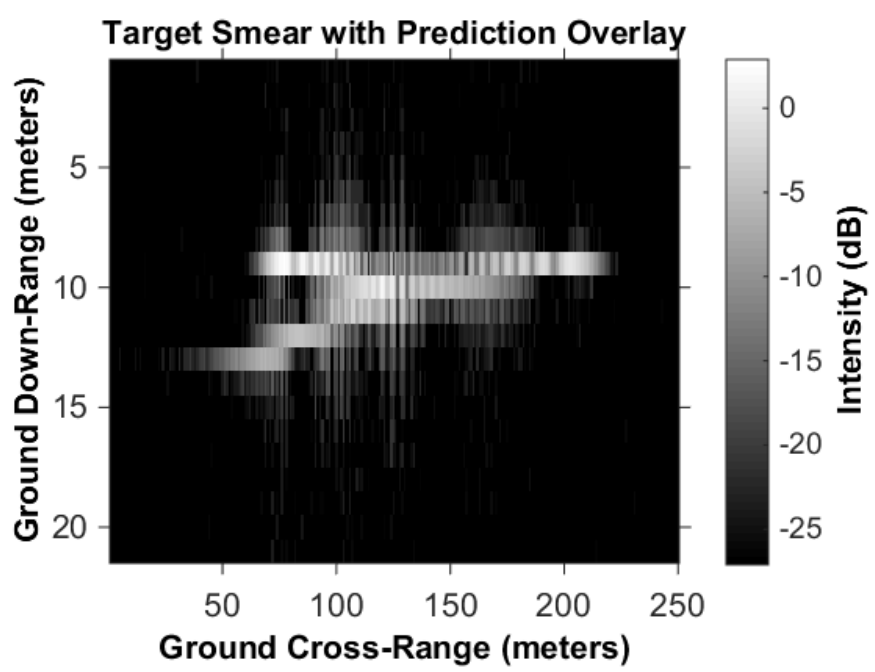

Fig. 2. Resulting target signature with $\left\{\bar{\alpha}_{0}=0.0 \mathrm{~m}\right\},\left\{\bar{\beta}_{0}=0.0 \mathrm{~m}\right\}$, $\left\{\bar{v}_{0}=9.62 \mathrm{~m} / \mathrm{s}\right\},\left\{\bar{w}_{0}=4.13 \mathrm{~m} / \mathrm{s}\right\},\left\{\bar{\gamma}_{0}=0.5 \mathrm{~s}\right\}$, and $\left\{\bar{\phi}_{0}=-81.3^{\circ}\right\}$ for the case of leftward pointing radar mainbeam

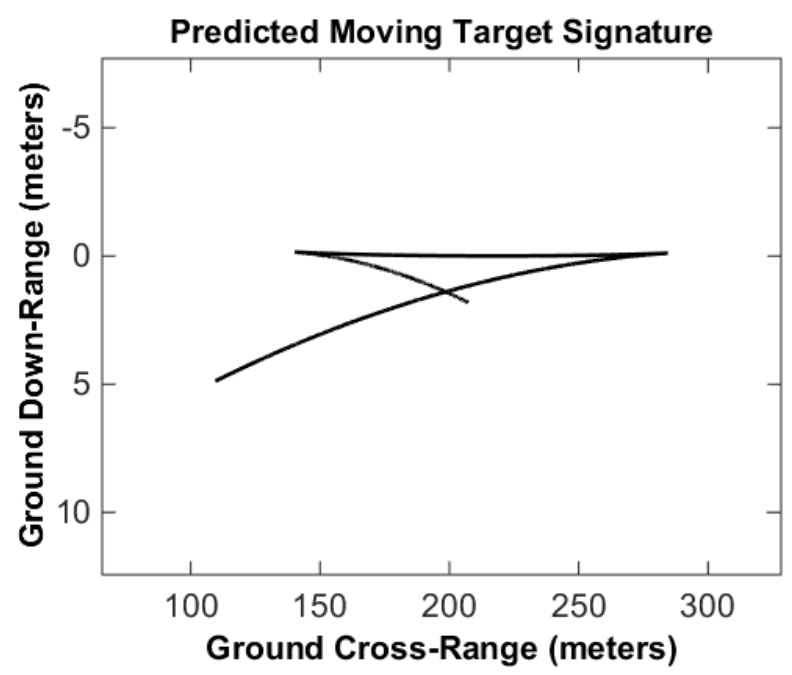

Fig. 3. Corresponding predicted target signature based upon the true target motion for the case of $\left\{\bar{\alpha}_{0}=0.0 \mathrm{~m}\right\},\left\{\bar{\beta}_{0}=0.0 \mathrm{~m}\right\},\left\{\bar{v}_{0}=9.62 \mathrm{~m} / \mathrm{s}\right\}$, $\left\{\bar{w}_{0}=4.13 \mathrm{~m} / \mathrm{s}\right\},\left\{\bar{\gamma}_{0}=0.5 \mathrm{~s}\right\}$, and $\left\{\bar{\phi}_{0}=-81.3^{\circ}\right\}$

The plots have similar parameters that were used to initially demonstrate complicated smear shapes within Ref. [9]. Such shapes exhibit more structure than the simply bowed smear shapes that are characteristic of targets moving with constant speed and heading. However, vehicle traffic in urban environments can undergo frequent maneuvers wherein the speed is increased, thus motivating the current research. In addition, this analysis of targets with increasing speed yields an accurate match between the predicted signatures of Figure 3 and the actual target signature of Figure 2. This analysis provides further verification of the predictive signature theory derived in Ref. [9].

Figures 2 and 3 present the SAR smears for the case of a radar mainbeam that is pointed off of the port (i.e., left) side of the radar platform. In contrast, Figures 4 and 5 compare the actual and predicted SAR smear signatures for the case of an identical target as with the previous example, but with 


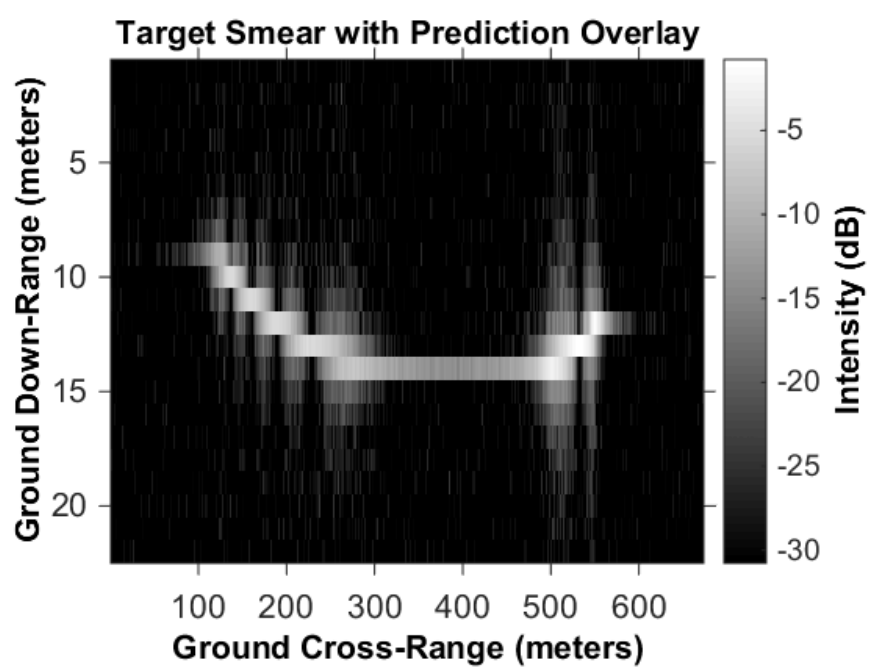

Fig. 4. Target signature in spotlight SAR imagery for a target with parameters $\left\{\bar{\alpha}_{0}=0.0 \mathrm{~m}\right\},\left\{\bar{\beta}_{0}=0.0 \mathrm{~m}\right\},\left\{\bar{v}_{0}=9.62 \mathrm{~m} / \mathrm{s}\right\},\left\{\bar{w}_{0}=4.13 \mathrm{~m} / \mathrm{s}\right\},\left\{\bar{\gamma}_{0}=\right.$ $0.5 \mathrm{~s}\}$, and $\left\{\phi_{0}=-81.3^{\circ}\right\}$ for the case of rightward pointing radar mainbeam

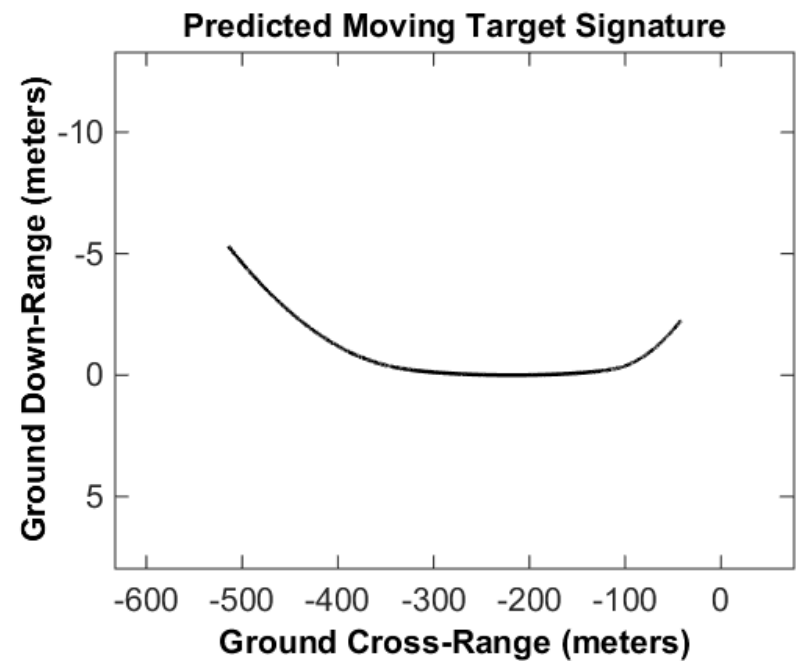

Fig. 5. Corresponding predicted signature for a target with $\left\{\bar{\alpha}_{0}=0.0 \mathrm{~m}\right\}$, $\left\{\vec{\beta}_{0}=0.0 \mathrm{~m}\right\},\left\{\bar{v}_{0}=9.62 \mathrm{~m} / \mathrm{s}\right\},\left\{\bar{w}_{0}=4.13 \mathrm{~m} / \mathrm{s}\right\},\left\{\bar{\gamma}_{0}=0.5 \mathrm{~s}\right\}$, and $\left\{\bar{\phi}_{0}=-81.3^{\circ}\right\}$ for the case of a rightward pointing radar mainbeam

the radar mainbeam is pointed off of the starboard (i.e., right) side of the radar platform. Again, there is excellent agreement between the predicted and actual SAR signatures in terms of smear position, extent, and shape. This case gives a different signature which almost crosses itself, which is quite different form the typical bowed signatures.

The next set of plots considers a target that is increasing speed, but with a significantly lower rate of acceleration in the direction opposite to that of the direction of motion, as modeled with the parameter set: $\left\{\bar{\alpha}_{0}=0.0 \mathrm{~m}\right\},\left\{\bar{\beta}_{0}=0.0 \mathrm{~m}\right\}$, $\left\{\bar{v}_{0}=9.62 \mathrm{~m} / \mathrm{s}\right\},\left\{\bar{w}_{0}=4.13 \mathrm{~m} / \mathrm{s}\right\},\left\{\bar{\gamma}_{0}=2.5 \mathrm{~s}\right\}$, and $\left\{\bar{\phi}_{0}=-81.3^{\circ}\right\}$. Only the parameter $\left\{\bar{\gamma}_{0}=2.5 \mathrm{~s}\right\}$ has a different value. Again, the increase in the target speed is modeled through the use of a hyperbolic tangent speed profile as provided in (9)-(10). These truth equations for the actual target motion are consistent with a speed profile of (11), as plotted in Figure 6.

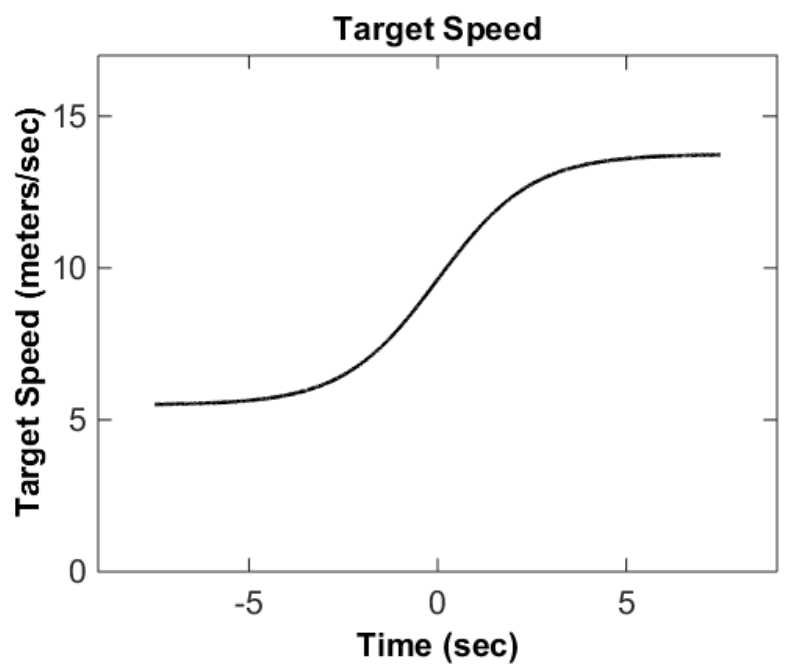

Fig. 6. Speed profile for a target that is increasing in speed according to the motion parameters $\left\{\bar{\alpha}_{0}=0.0 \mathrm{~m}\right\},\left\{\bar{\beta}_{0}=0.0 \mathrm{~m}\right\},\left\{\bar{v}_{0}=9.62 \mathrm{~m} / \mathrm{s}\right\}$, $\left\{\bar{w}_{0}=4.13 \mathrm{~m} / \mathrm{s}\right\},\left\{\bar{\gamma}_{0}=2.5 \mathrm{~s}\right\}$, and $\left\{\bar{\phi}_{0}=-81.3^{\circ}\right\}$

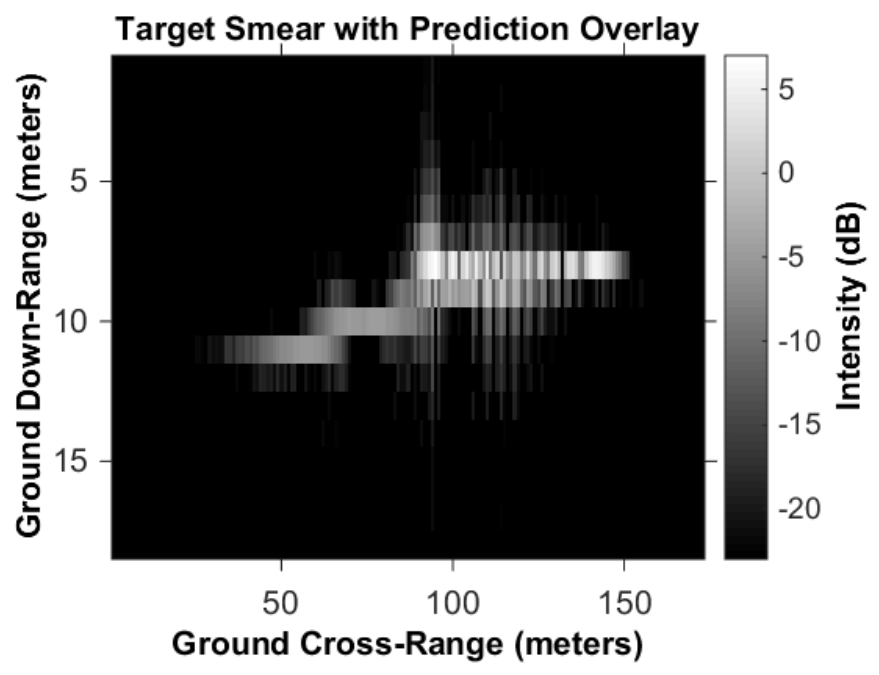

Fig. 7. Resulting target signature with $\left\{\bar{\alpha}_{0}=0.0 \mathrm{~m}\right\},\left\{\bar{\beta}_{0}=0.0 \mathrm{~m}\right\}$, $\left\{\bar{v}_{0}=9.62 \mathrm{~m} / \mathrm{s}\right\},\left\{\bar{w}_{0}=4.13 \mathrm{~m} / \mathrm{s}\right\},\left\{\bar{\gamma}_{0}=2.5 \mathrm{~s}\right\}$, and $\left\{\bar{\phi}_{0}=-81.3^{\circ}\right\}$ for the case of leftward pointing radar mainbeam

Figures 7 and 8 present the SAR smears for the case of a radar mainbeam that is pointed off of the port (i.e., left) side of the radar platform. This smear signature has a gentler curve than that of the faster braking case given above.

Figures 9 and 10 compare the actual and predicted SAR smear signatures for the case of a slow rate of speed increase than with the previous example, but with the radar mainbeam is pointed off of the starboard (i.e., right) side of the radar platform. Again, there is excellent agreement between the predicted and actual SAR signatures in terms of smear position, extent, and shape. This case gives a different signature which almost crosses itself, just as for the case of a braking target with a larger value for the acceleration in the direction opposite to that of the direction of motion. 


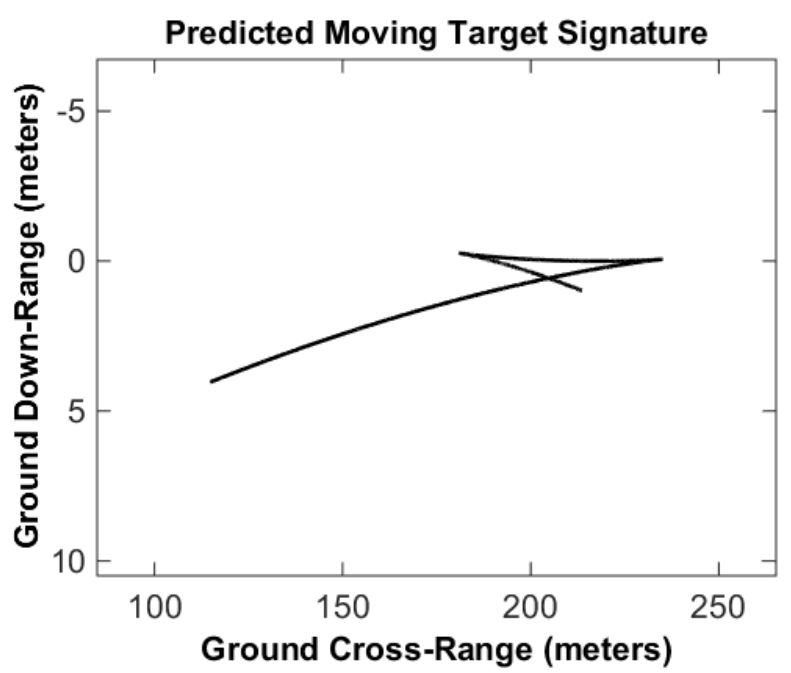

Fig. 8. Corresponding predicted target signature based upon the true target motion for the case of $\left\{\bar{\alpha}_{0}=0.0 \mathrm{~m}\right\},\left\{\bar{\beta}_{0}=0.0 \mathrm{~m}\right\},\left\{\bar{v}_{0}=9.62 \mathrm{~m} / \mathrm{s}\right\}$, $\left\{\bar{w}_{0}=4.13 \mathrm{~m} / \mathrm{s}\right\},\left\{\bar{\gamma}_{0}=2.5 \mathrm{~s}\right\}$, and $\left\{\bar{\phi}_{0}=-81.3^{\circ}\right\}$

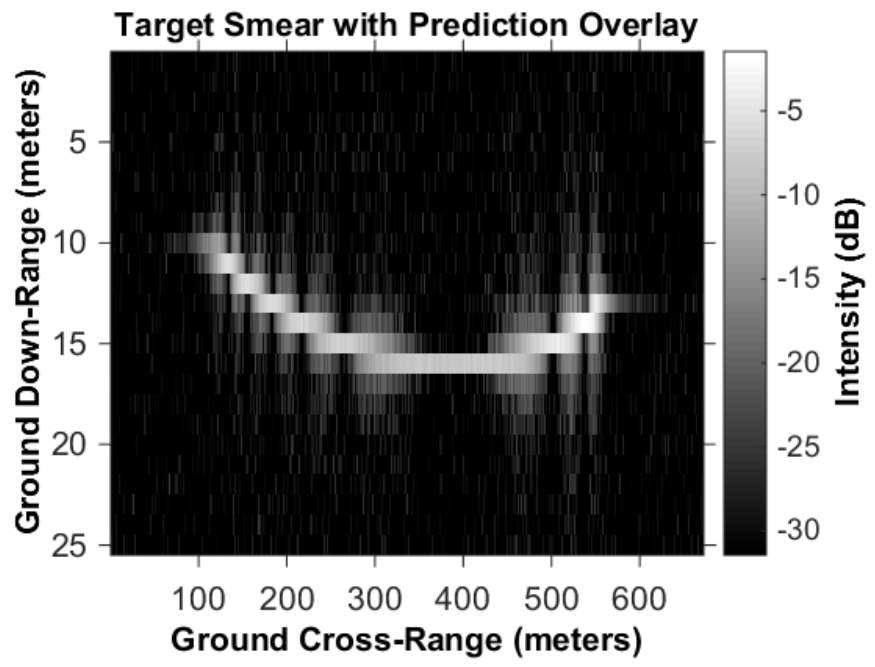

Fig. 9. Target signature in spotlight SAR imagery for a target with parameters $\left\{\bar{\alpha}_{0}=0.0 \mathrm{~m}\right\},\left\{\bar{\beta}_{0}=0.0 \mathrm{~m}\right\},\left\{\bar{v}_{0}=9.62 \mathrm{~m} / \mathrm{s}\right\},\left\{\bar{w}_{0}=4.13 \mathrm{~m} / \mathrm{s}\right\},\left\{\bar{\gamma}_{0}=\right.$ $2.5 \mathrm{~s}\}$, and $\left\{\phi_{0}=-81.3^{\circ}\right\}$ for the case of rightward pointing radar mainbeam

\section{CONCLUSION}

This paper has examined the signatures of surface moving targets that are increasing in speed during the SAR collection interval. Consistent signatures smears were obtained from both the application of analytic predictive equations and the generation using SAR simulations. It was found that the resulting signature shapes need not conform to conventional bow-shaped morphologies. In particular, this analysis compared signature of targets with a higher rate of speed increase versus those with a slower rate. For both sets of cases, similarly interesting and complicated target signature smears result.

\section{ACKNOWLEDGMENT}

DoD Distribution Statement A: Unlimited Distribution. The views expressed in this document are those of the authors and do not reflect the official policy or position of the Department of Defense or the U.S. Government.

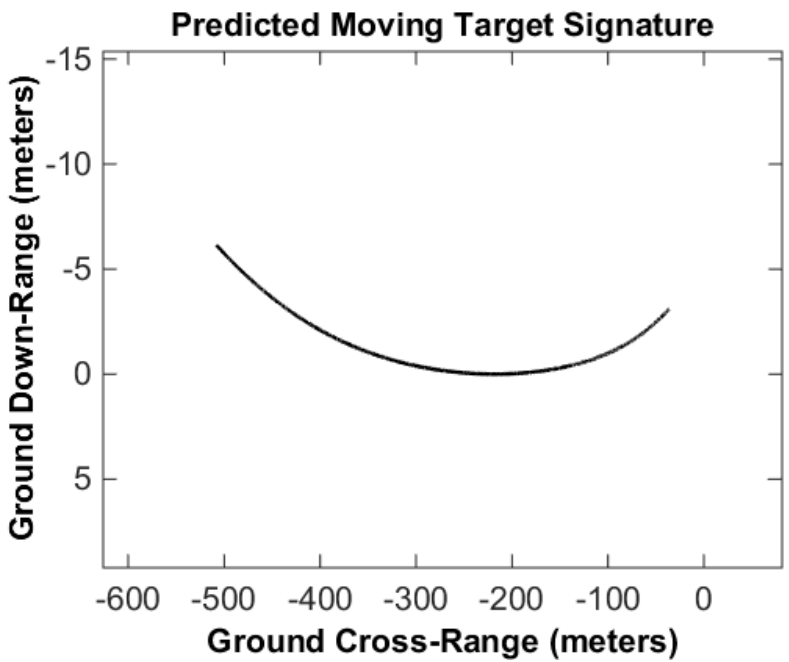

Fig. 10. Corresponding predicted signature for a target with $\left\{\bar{\alpha}_{0}=0.0 \mathrm{~m}\right\}$, $\left\{\bar{\beta}_{0}=0.0 \mathrm{~m}\right\},\left\{\bar{v}_{0}=9.62 \mathrm{~m} / \mathrm{s}\right\},\left\{\bar{w}_{0}=4.13 \mathrm{~m} / \mathrm{s}\right\},\left\{\bar{\gamma}_{0}=2.5 \mathrm{~s}\right\}$, and $\left\{\bar{\phi}_{0}=-81.3^{\circ}\right\}$ for the case of a rightward pointing radar mainbeam

\section{REFERENCES}

[1] R. P. Perry, R. C. DiPietro, and R. L. Fante, "SAR imaging of moving targets," IEEE Transactions on Aerospace and Electronic Systems, vol. 35, no. 1, pp. pp. 188-200, Jan 1999.

[2] R. K. Raney, "Synthetic aperture imaging radar and moving targets," IEEE Transactions on Aerospace and Electronic Systems, vol. 7, no. 3, pp. pp. 499-505, May 1971.

[3] J. K. Jao, "Theory of synthetic aperture radar imaging of a moving target," IEEE Transactions on Geoscience and Remote Sensing, vol. 39, no. 9, pp. $1984-1992$, Sep 2001.

[4] R. H. Stolt, "Migration by fourier transform," Geophysics, vol. 43, pp. 23-48, 1978

[5] S. Rahman, "Focusing moving targets using range migration algorithm in ultra wideband low frequency synthetic aperture radar," Master's thesis, Blekinge Institue of Technology, Jun 2010.

[6] D. Garren, J. W. Scrofani, M. Tummala, and J. C. McEachen, "Target migration path morphology of moving targets in spotlight sar," Proc. of SPIE Vol. 8746 87460B-7: "Algorithms for Synthetic Aperture Radar Imagery XX" of the SPIE Defense, Security, and Sensing Conference held 29 April - 3 May 2013 in Baltimore, Maryland; USA, May 2013.

[7] C. V. Jakowatz Jr., D. E. Wahl, P. H. Eichel, D. C. Ghiglia, and P. A.Thompson, Spotlight-Mode Synthetic Aperture Radar: A Signal Processing Approach. Norwell, MA: Kluwer Academic, 1996.

[8] W. G. Carrara, R. S. Goodman, and R. M. Majewski, Spotlight Synthetic Aperture Radar Signal Processing Algorithms. Norwood, MA, USA: Artech House, 1995.

[9] D. A. Garren, "Smear signature morphology of surface targets with arbitrary motion in spotlight synthetic aperture radar imagery," IET Radar, Sonar and Navigation, vol. 8, no. 5, pp. 435-448, Jun 2014.

[10] J. Zhang, J. Xu, Y. Peng, and X. Wang, "Speckle filtering of SAR images based on sub-aperture technique and principal component analysis," Communications and Information Technology 2005. ISCIT 2005. IEEE International Symposium on, vol. 2, pp. 1217-1222, Oct 2005.

[11] M. Soumekh, "A system model and inversion for synthetic aperture radar imaging," IEEE Transactions on Image Processing, vol. 1, no. 1, pp. 64-76, Jan 1992.

[12] _ "Digital spotlighting and coherent subaperture image formation for stripmap synthetic aperture radar," Image Processing, 1994, Proceedings, ICIP-94, IEEE International Conference, vol. 1, pp. 476-480, Nov 1994.

[13] D. A. Garren, "Signatures of braking surface targets in spotlight synthetic aperture radar," Proceedings of 2014 Sensor Signal Processing for Defence, held in Edinburgh, UK, on 08-09 September 2014, pp. 5155, Sep 2014. 